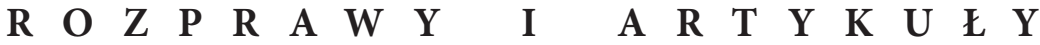

Ius Matrimoniale

26 (2015) nr 1

Mons. Giordano Caberletti

Tribunale della Rota Romana

\section{Józef Florczak: Prelato Uditore della Rota Romana morto nel Lager Dachau. \\ Parte I}

Solum Deum prae oculis habentes: sono le parole sommamente impegnative scritte nella parte finale di una sentenza, prima che vengano apposte le firme dei giudicanti.

Sicuramente a Mons. Józef Florczak era familiare questa formula, ma soprattutto egli ha dimostrato di averla scritta con la sua vita nel fedele servizio al Vangelo sulla cattedra universitaria, nelle aule del Tribunale della Rota Romana, fra il popolo cristiano della sua parrocchia, fino a farla risplendere con la testimonianza del suo sacrificio nella notte di un lager nazista.

Se è vero che il Tribunale della Rota Romana forma la giurisprudenza che guida tutti i Tribunali della Chiesa nell'esercizio della giustizia, non si possono dimenticare le persone che hanno dato intelligenza e cuore a questo Tribunale Apostolico e pertanto è giusto ricordare uno dei suoi Prelati che, oltre settant'anni fa, è stato privato della libertà e della vita da un sistema tra i più iniqui.

Da questa considerazione nasce l'idea di presentare la figura di Mons. Florczak, illustre Prelato Uditore, tenuto anche presente, come si vedrà nel prosieguo di questo testo, che il suo contributo al diritto matrimoniale canonico, applicato nel foro canonico negli anni '20 del secolo scorso, è anche oggi di indubbio valore. 


\section{Cenni biografici}

Mons. Józef Florczak, prima di consumare eroicamente la sua vita a Dachau, servì la Chiesa come docente universitario a Lublino, come giudice del Tribunale Apostolico della Rota Romana, come parroco in una piccola città della Polonia ${ }^{1}$.

Era nato il 25 settembre 1885 in Luciejów, diocesi di Wladislavia (Włocławek) ${ }^{2}$. Nel settembre dell'anno 1903 entrò nel seminario di Włocławek. Dopo essere stato ordinato suddiacono, nell'autunno del 1909 compì ulteriori studi teologici all'Accademia di San Pietroburgo. Venne consacrato presbitero il 7 agosto 1910. Nel 1913 conseguì il titolo di magister theologiae. Fu inviato a Roma per gli studi giuridici, da lui compiuti negli anni 1913-1914³ al Pontificium Collegium

1 Per le notizie biografiche relative a Mons. Józef Florczak cfr.: K. OrzEszyna, Józef Florczak (1887-1943), in Profesorowie prawa Katolickiego Uniwersytetu Lubelskiego, a cura di A. Dęвiński - W. Sz. Staszewski - M. WójCik, Lublin 2008, p. 135-138; K. RulKA, v. Florczak Józef, in Encyklopedia Katolicka, t. 5, Lublin 1989, col. 329; K. RulKa, v. Florczak Józef, in Włocławski słownik biograficzny, a cura di S. Kunikowski, vol. VI, Włocławek 2011, p. 29-31. Ringrazio vivamente per la traduzione di questi contributi Mons. Grzegorz Erlebach, Prelato Uditore della Rota Romana, e Mons. Robert Gołębiowski, Difensore del Vincolo nel Tribunale della Rota Romana.

Un profilo biografico di Mons. Józef Florczak venne riferito nel necrologio del Bollettino Ufficiale del Vicariatus Urbis del settembre 1949 (cfr. Bollettino del Clero Romano. Ufficiale per gli Atti del Vicariato 30 [1949], p. 139-140).

2 Questa data e questo luogo sono riferiti in Annuario Pontificio per l'anno 1922, Roma 1922, p. 430, e a partire da questo fino all'Annuario Pontificio del 1928 (l'ultimo in cui Mons. Florczak è indicato come Prelato Uditore della Rota), la data della sua nascita è 25 settembre 1885, mentre nell'Annuario Pontificio del 1921, alla p. 421, risulta che Mons. Florczak era nato il 25 settembre 1887; l'articolo di K. Orzeszyna, citato nella nota precedente, alla p. 135 indica come data di nascita il 25 settembre 1887 e come luogo di nascita Brzyków (comune di Łask); la stessa data e lo stesso luogo, indicati da K. Orzeszyna, sono riportati anche da K. Rulka nell'articolo citato alla suddetta nota (K. Rulka precisa che Brzyków si trova vicino a Sieradz). Ritengo più attendibili i dati riferiti dall'Annuario Pontificio a partire dall'anno 1922 fino al 1928, in quanto certamente sono stati controllati da Mons. Florczak.

3 Nell'elenco degli studenti iscritti per l'anno accademico 1917-1918 all'Angelicum "Josephus Florzack [sic!]” risulta "Doct. in J. C.”. 
Internationale "Angelicum", laureandosi con la tesi dal titolo De caelibatu cleri Poloniae.

Nel 1918 venne nominato professore all'Università cattolica di Lublino, dove insegnò per due anni accademici (1918-1919, 1919-1920) nella Facoltà di Diritto Canonico e di Scienze Morali. La sua attività didattico-scientifica riguardava la storia del diritto canonico e le norme generali del Codex Iuris Canonici; nell'anno accademico 19191920 condusse anche un seminario su "Impedimenti matrimoniali alla luce delle decisioni della Rota Romana e della Congregazione dei Sacramenti". Nella Facoltà di Diritto e di Scienze Sociali-Economiche insegnò diritto ecclesiastico. Fu eletto decano della Facoltà di Diritto Canonico e di Scienze Morali per l'anno accademico 1919-1920.

Il 3 luglio 1919 presso il Pontificium Collegium Internationale "Angelicum" Józef Florczak si laureò in S. Teologia5.

All'età di soli 35 anni, il 16 febbraio 1920 venne nominato da Benedetto XV Prelato Uditore del Tribunale della Rota Romana ed in questo ufficio rimase fino a 15 ottobre $1929^{6}$. Mons. Florczak si stabilì pertanto a Roma, dove nel 1920, nominato dal Card. A. S.

${ }^{4}$ Cfr. K. Rulka, Florczak Józef, in Włocławski słownik biograficzny, cit., p. 31.

5 Questo è il verbale della sessione di laurea, sottoscritto da fr. Alberto Blat, O.P. et da fr. Eduardo Hugon, O.P., Pro-Reggente: "Die 3 Julii 1919, R. D. Mag. et Dr. Juris Can. Josephus Florczak, sacerdos dioecesis Wladislaviensis subiit examen Doctoratus Sacrae Theologiae. Eius Dissertatio «De coelibatu clericorum in Polonia» fuit approbata a PP. Pro-Regente et Pègues maxima cum laude. In examine orali, habuit in primo scrutinio vota alba 5 ex 5; et in secundo 20 ex 25. Examinatores fuerunt RR. PP. Pro-Regens, Pègues, Blanco, Garrigou et Vosté. Die autem 4 Julii, servatis servandis, per R. P. Pro-Regentem fuit rite promotus, ac litterae testimoniales ipsi traditae sunt".

Ringrazio il R. P. Miroslav Konstanc Adam, O.P., Rettore della Pontificia Università S. Tommaso d'Aquino, per aver permesso al sig. M. Candelaresi, della Segreteria dell'Angelicum, di fornirmi copia di questo atto e copia dell'elenco degli studenti dell'anno accademico 1917-1918.

${ }^{6}$ Queste notizie sono in S. Killermann, Die Rota Romana. Wesen und Wirken des päpstlichen Gerichtshofes im Wandel der Zeit, Peter Lang, Frankfurt am MainBerlin-Bern-Bruxelles-New York-Oxford-Wien 2009, p. 430-431. 
Sapieha, divenne anche Rettore della chiesa di S. Stanislao, chiesa nazionale dei Polacchi.

Fu nominato consultore della S. Congregazione dei Sacramenti ${ }^{7}$.

Il 16 maggio 1922 nell'Aula Magna dell'Università di Padova, insieme a tutti i responsabili delle delegazioni straniere convenute a Padova per celebrare il settimo centenario dell'Università, a Mons. Florczak, che rappresentava l'Università cattolica di Lublino, fu conferita la laurea ad honorem ${ }^{8}$.

Il 3 gennaio 1925, riconoscendo i suoi meriti acquisiti in Polonia ed all'estero, il vescovo Stanisław Zdzitowiecki, nominò Mons. Florczak canonico onorario della collegiata di Kalisz. Un po' più tardi il vescovo Henryk Przeździecki lo inserì tra i canonici onorari della cattedrale di Siedlce.

Il 27 ottobre 1931 Mons. Florczak tornò in Polonia ${ }^{9}$ e il Card. Alexander Kakowski, Arcivescovo di Varsavia, sostenuto dal Card. Augusto Hlond, Arcivescovo di Gniezno e Primate di Polonia, si preoccupò di garantirgli una dignitosa collocazione, come risulta

7 Nell'Annuario Pontificio del 1935 (p. 653) per l'ultima volta appare come consultore della S. Congregazione della disciplina dei Sacramenti, e solo lui è indicato come residente fuori Roma.

8 Ringrazio il prof. Francesco Piovan, dell'Università di Padova, che, su interessamento del prof. Giampaolo Romanato, della stessa Università, gentilmente mi ha fornito questa notizia, reperibile in Acta Universitatis Patavinae septima saecularia celebrantis MCCXXII-MCMXXII, Tipografia Antoniana, Padova 1925, p. 119.

9 Cfr. I «Fogli di Udienza» del cardinale Eugenio Pacelli Segretario di Stato, vol. II (1931), a cura di G. Coco - A. M. Dieguez, Collectanea Archivi Vaticani 95, Città del Vaticano - Archivio Segreto 2014, p. 492, n. 2984.

Non è chiaro il motivo della rinuncia di Mons. Florczak all'ufficio del giudice della Rota. Sembra che ciò sia stato originato da qualche accusa, mossa nei suoi confronti - a quanto pare riguardo all'osservanza del segreto professionale -, di cui fondatezza non è stata dimostrata. Risulta infatti che il Card. A. Kakowski, Arcivescovo di Varsavia, che aveva proposto Mons. Florczak al S. Padre Benedetto XV per l'ufficio di Uditore della Rota Romana, con lettera del 20 aprile 1928 indirizzata a Mons. Florczak, pur riconoscendo che "Il Supremo Tribunale della Segnatura emise la sentenza, che non era provata la violazione formale del segreto professionale imputata a Monsignor Florczak" (Archivio Segreto Vaticano, Arch. Nunz. Varsavia, 254, fasc. 29), lo invitò a dimettersi a motivo di qualche imprudenza da lui commessa. 
dalla corrispondenza intercorsa tra i due cardinali polacchi, il Card. Pietro Gasparri, Segretario di Stato, Mons. Francesco Marmaggi, Nunzio Apostolico in Polonia, e Mons. Massimo Massimi, Decano della Rota ${ }^{10}$.

A Mons. Florczak, oltre alla cattedra di diritto canonico all'Università di Varsavia, oppure all'Università di Lublino, fu proposto l'insegnamento di diritto canonico al Grande Seminario Polacco di Detroit, ma nessuna di queste possibilità ebbe esito. Al ritorno nella sua diocesi di Włocławek, Mons. Florczak venne nominato parroco di Turek, e qui egli dimostrò instancabile carità pastorale, contribuendo all'edificazione del ginnasio e della Casa Cattolica. Quest'ultima doveva servire a tutti i parrocchiani come luogo per incontri, recite e conferenze ${ }^{11}$. Egli fece dipingere la chiesa parrocchiale, dedicata al SS. Cuore di Gesù e la fece ornare di vetrate, opera del prof. Józef Mehoffer ${ }^{12}$, uno dei principali esponenti del movimento della Giovane Polonia. Il 3 dicembre 1936 divenne decano del decanato di Turek.

Le onorificenze ricevute da Mons. Florczak dimostrano la grande considerazione di cui egli godeva nella Chiesa e nella società polacca. Con Breve pontificio fu nominato Prelato domestico di Sua Santità ${ }^{13}$ e Prelato Arcidiacono della basilica di Włocławek, mentre il governo polacco gli conferì la Croce commendatoriale Polonia Restituta, riconoscendo quanto aveva compiuto per il bene della Patria.

Come per migliaia di sacerdoti polacchi, il ministero parrocchiale di Mons. Florczak venne drammaticamente interrotto dal furore nazista.

\footnotetext{
Notizie sulle dimissioni di Mons. Florczak da Prelato Uditore della Rota sono riportate anche in $I$ «Fogli di Udienza» del cardinale Eugenio Pacelli Segretario di Stato, vol. II (1931), cit., p. 491-492.

${ }_{10}$ Cfr. Archivio Segreto Vaticano, Arch. Nunz. Varsavia, 254, fasc. 29.

11 Fonte di queste informazioni è: www.mipbp.turek.net.pl/Florczak.html, 2012.

12 Le notizie relative alla chiesa di Turek si trovano in http://12u.pl/u/Details, asp $\mathrm{x}$ ?mik=1\&id=C===36\&mas=96, 3.08.2014.

13 Venne nominato l'8 novembre 1919 e appare nell'elenco dei Prelati domestici anche nell'Annuario Pontificio del 1943 (p. 796).
} 
I principi anticristiani del nazismo, condannati con fermezza da Pio XI nell'enciclica Mit brennender Sorge del 14 marzo $1937^{14}$, e coraggiosamente contrastati dai vescovi tedeschi Card. Michael von Faulhaber, arcivescovo di Monaco e Frisinga, Mons. Clemens von Galen, vescovo di Münster, Mons. Konrad von Preysing, vescovo di Berlino $^{15}$, in Polonia furono applicati con un odio senza limiti, in particolare nei confronti della Chiesa Cattolica ${ }^{16}$.

${ }_{14}$ Cfr. AAS 29 (1937), p. 145-167; tr. it., ibid., p. 168-188. Per le posizioni di Pio XI nei confronti di Hitler e del nazismo cfr.: Y. ChIron, Pio XI. Il papa dei Patti Lateranensi e dell'opposizione ai totalitarismi, tr. it., San Paolo, Milano 2006, p. 342-375, 426-435, 445-451.

15 Per la resistenza della Chiesa Cattolica in Germania al nazismo cfr. L. GARIBALDI, O la croce o la svastica. La vera storia dei rapporti tra la Chiesa e il nazismo, Lindau, Torino 2009. Grande testimone di opposizione al nazionalsocialismo è stato il beato Rupert Mayer, S.I. (R. HAUB, Rupert Mayer. L'impegno per la Verità, tr. dal tedesco, Edizioni AdP, Roma 2005).

16 Sull'immane sofferenza subita dalla Polonia, ed in particolare dalla Chiesa Polacca, durante l'occupazione nazista cfr. L. Müllerova - A. StAniwski, Gli anni della guerra e dell'occupazione (1939-1945), in Storia del cristianesimo in Polonia, a cura di J. KŁoczowski, tr. it., CSEO, Bologna 1980, p. 443-471; L. Müllerova, La Chiesa polacca dal 1939 ai nostri giorni, in Storia religiosa della Polonia, a cura di L. VAcCAro, La Casa di Matriona e Fondazione Ambrosiana Paolo VI, Milano 1985, 205-212; A. RiCCARDI, Il secolo del martirio. I cristiani nel Novecento, Oscar Mondadori, Milano 2000, p. 87-99; P. BLET, Pio XII y la segunda guerra mundial, tr. dal francese, Ediciones Cristianidad, Madrid 20132, p. 103-134. Nella sua prima enciclica Summi Pontificatus (20 ottobre 1939) Pio XII con forza alzò la sua voce paterna per protestare contro l'invasione della Polonia: "Dum [...] has lineas exaramus terrificus affertur Nobis nuntius nefandum iam belli incendium, quod enixe deprecari conati sumus, miserrime conflatum esse [...]. Summa aegritudine paternus oppletur animus, dum ea omnia mente prospicimus, quae ex tenebricoso violentiae simultatisque semine oritura sunt, cui iam gladius cruentos facit sulcos [...]. Atque tot hominum cruor, eorum etiam, qui, exercitus ordinibus non adsciti, misere occubuere, lugubrem videtur gemitum ex dilecta praesertim ea natione extollere, ex Polonia dicimus, quae ob tenacem suam erga Ecclesiam fidelitatem, itemque ob praeclara in christianum tutandum civilemque cultum promerita, historiae fastis inscripta immortalitatique commendata, humanam fraternamque iure meritoque postulat ab omnibus commiserationem" (AAS 31 [1939], p. 421, 449). 
Hitler voleva 1"“annientamento del popolo polacco"17. Il 6 aprile 1941, il Presidente della Polonia Władysław Raczkiewicz dal suo esilio di Londra scrisse a Pio XII per informarlo del "martirio della Polonia"18.

Il Bollettino della Diocesi di Roma riferisce queste notizie precise sulla via crucis di Mons. Florczak: "Scoppiata la guerra, nell'ottobre del 1939 egli si trovava a Varsavia, dove i tedeschi lo trassero prigioniero, insieme ad altri sacerdoti, al villaggio di Pawiak; liberato dopo alcune settimane, fu nuovamente imprigionato nel 1942, e tradotto nel Campo di Concentramento di Oswiecim, e subito dopo a quello di Dachau, di tremenda memoria; era contrassegnato col numero 30277. Nella primavera del 1943 fu colpito da grave infiammazione di polmoni, trasportato all'ospedale del detto campo, e qui, assistito da [...] Mons. Francesco Korsynki [...] di Wladislavia, e dai Canonici Biskupoki [sic!] e Kunca, condiocesani, e compagni tutti e tre di prigionia, il 20 aprile 1943 rese l'anima a Dio, pienamente rassegnato alla divina volontà e pieno di fiducia nella bontà del Signore"19.

Il suo corpo fu bruciato nel forno crematorio del Lager.

Valgono sicuramente anche per Mons. Florczak le parole pronunciate da S. Giovanni Paolo II a Varsavia il 13 giugno 1999 nell'omelia per la beatificazione di 108 martiri polacchi, il cui sacrificio si consumò a causa dell'odio nazista durante la seconda guerra mondiale: "le più dure prove non erano in grado di distoglierci dall'amore di Dio [...]. I beati martiri gridano ai nostri cuori: Credete che Dio è amore! Credetelo nel bene e nel male" ${ }^{20}$.

\footnotetext{
17 G. Angelozzi Gariboldi, Il Vaticano nella seconda guerra mondiale, Milano 1992, p. 186.

18 Ibid., p. 198.

19 Bollettino del Clero Romano. Ufficiale per gli Atti del Vicariato 30 (1949), p. 139-140.

20 L'Osservatore Romano, 14-15 giugno 1999, anno 139, n. 135, p. 8-9; il testo dell'omelia in lingua polacca è in Insegnamenti di Giovanni Paolo II, XXII, 1 (1999, gennaio-giugno), Città del Vaticano 2002, p. 1326-1333.
} 


\section{Ricerca scientifica}

È stato osservato che l'attività scientifica di Mons. Florczak fu piuttosto modesta ${ }^{21}$. Questo limite forse è dovuto al breve periodo di insegnamento universitario, oppure alla successiva dedizione all'ufficio di giudice rotale.

Mons. Florczak pubblicò solo tre articoli ${ }^{22}$, che hanno come oggetto le vicende storiche del celibato ecclesiastico in Polonia ${ }^{23}$. Probabilmente, visto il titolo della sua tesi di laurea in diritto canonico all'Angelicum e considerato che alla fine di ogni articolo pone la voce "Roma", si tratta dello stesso testo della tesi.

Anche se i tre articoli sono piuttosto brevi (complessivamente arrivano a cinquantasei pagine), si coglie immediatamente in ognuno di essi un ricco apparato critico, che prova uno studio meticoloso. Poiché la ricerca parte dal sec. XIII ed arriva fino al Concilio Vaticano I, è da apprezzare la capacità di sintesi dimostrata dall'autore.

Mons. Florczak inizia ricordando le circostanze in cui il cristianesimo fu portato in Polonia. Poiché i primi missionari arrivati in Polonia erano monaci, e quindi celibi, sebbene in quel momento il Papato non risplendesse per virtù, la testimonianza dei primi missionari fece sicuramente apprezzare il valore della castità perfetta ${ }^{24}$. Dopo che gli abusi avevano offuscato il comportamento dei chierici, iniziò da Cluny la riforma, partendo dunque dal basso, come osserva acutamente Florczak ${ }^{25}$. Sarà Gregorio VII ad avviare una

\footnotetext{
21 Cfr. K. Orszyna, cit., p. 137.

22 K. Rulka scrive che quei tre articoli riprendono, o addirittura sono, i capitoli della tesi dottorale (cfr. scheda citata alla nota n. 4).

23 1) Celibat duchowieństwa $w$ Polsce do XIII w. Studium historyczno-prawne, Ateneum Kapłańskie 2 (1914), z. 1-2 (51-52), p. 49-59, 147-151); 2) Celibat duchowieństwa polskiego w XIII w. Studium historyczno-prawne, Ateneum Kapłańskie 2 (1914), z. 3 (53), p. 211-224; 3) Celibat duchowieństwa w Polsce. I. Czasy Reformacji II. Od przyjęcia w Polsce postanowień Soboru Trydenckiego do czasów ostatnich, Ateneum Kapłańskie 2 (1914), z. 6 (56), p. 17-41. Ha tradotto questi articoli il dott. G. Sandhof.

${ }^{24}$ Cfr. J. Florczak, Celibat..., Ateneum Kapłańskie 2 (1914), z.1, p. 51.

25 Cfr. ibid., p. 52.
} 
vasta e profonda riforma della Chiesa, che aveva tra gli obiettivi pure quello di portare i chierici all'osservanza del celibato ${ }^{26}$. La riforma gregoriana raggiunse anche la Polonia, dove si era diffusa la clerogamia $^{27}$. Già Gregorio VII, Sommo Pontefice dal 1073 al 1085, aveva dimostrato la sua sollecitudine per la Polonia scrivendo il 24 aprile 1075 una lettera a Boleslao II e impegnandosi a ripristinare il celibato ecclesiastico con l'invio di suoi legati ${ }^{28}$.

Se l'inviato pontificio "Walon", vescovo di Beauvais, zelante sostenitore delle idee gregoriane, fu costretto a destituire dal loro ufficio due vescovi della provincia di Gniezno a motivo della simonia, c'è da pensare che egli non abbia tralasciato di affrontare gli abusi nei confronti del celibato ecclesiastico ${ }^{29}$. Successivamente Callisto II, Papa dal 1119 al 1124, inviò come suo legato in Polonia il Card. Gilles, vescovo di Tusculum, che indisse un sinodo, ed è da credere che la questione del celibato non sia stata omessa ${ }^{30}$.

Il grande spirito delle riforme proveniente da Roma al tempo di Alessandro III (1159-1181), si è fatto sentire anche in Polonia, dove a Łęczyca nel $1180 \mathrm{fu}$ convocato un sinodo, che non toccò, a quanto sembra, il celibato ecclesiastico, ma non perché in quel tempo la disciplina in Polonia fosse osservata. Di fatto in Polonia i grandi sforzi della riforma iniziata con Gregorio VII non si sono quasi mai avvertiti, forse anche perché l'opera degli inviati pontifici non poteva essere decisiva $^{31}$. Nell'elenco di benefattori di monasteri o di chiese vengono infatti ricordati figli o concubine di sacerdoti ${ }^{32}$.

Il Card. Pietro di Capua, legato di Celestino III (1191-1198), indisse due sinodi e visitò quasi tutta la diocesi di Cracovia, presentando ovunque il celibato come obbligo da osservare. Il clero accolse con grande difficoltà le leggi che ordinavano l'interruzione di legami

\footnotetext{
6 Cfr. ibidem.

Cfr. ibid., p. 54.

Cfr. ibid., p. 53-54.

Cfr. ibid., p. 55.

${ }^{30}$ Cfr. ibid., p. 55-56.

31 Cfr. ibid., p. 57.

32 Cfr. ibid., p. 57-58.
} 
illeciti, da cui spesso erano nati figli. Il ripristino del celibato ha avuto un percorso lungo, non bastando interventi temporanei, anche se forti, come erano quelli degli inviati pontifici ${ }^{33}$.

Florczak critica come non fondata l'affermazione, diffusa da alcuni studiosi polacchi ${ }^{34}$, secondo la quale ci furono in Polonia anche vescovi sposati ${ }^{35}$.

Nel secondo articolo Florczak evidenzia come la grande opera riformatrice di Innocenzo III (1198-1216) ${ }^{36}$ abbia raggiunto la Polonia. Fu l'arcivesco Henryk Kietlicz (1199-1219), autorevole guida spirituale, a lottare contro i vizi del clero ${ }^{37}$. Egli stesso si recò a Roma per informare il Pontefice sul triste stato morale del clero polacco e lo stesso Innocenzo III con il breve "Cum decorem domus Domini" del 5 gennaio 1206 si rivolse ai vescovi polacchi chiedendo loro di ripristinare il celibato ecclesiastico e di rimuovere dalla Chiesa tutti gli abusi e i comportamenti scandalosi ${ }^{38}$; tra altri abusi, avveniva che figli di canonici pretendevano di ereditare la prebenda del padre ${ }^{39}$. Larcivescovo Kietlicz fece di tutto per porre fine al concubinato dei preti $^{40}$, in particolare con il sinodo convocato nel luglio 1210 a Borzyków ${ }^{41}$. Anche Pełka, arcivescovo di Łęczyca, si adoperò intensamente per la riforma dei costumi del clero $^{42}$.

Al sinodo di Breslavia, svoltosi il 10 ottobre 1248 su convocazione del legato di Innocenzo IV (1243-1254), l'arcidiacono di Liegi Giacomo di Pantaleon, sono state prese decisioni riguardanti il celibato ecclesiastico ${ }^{43}$. Poi, nel 1251, nel sinodo di Poznań, convocato dal

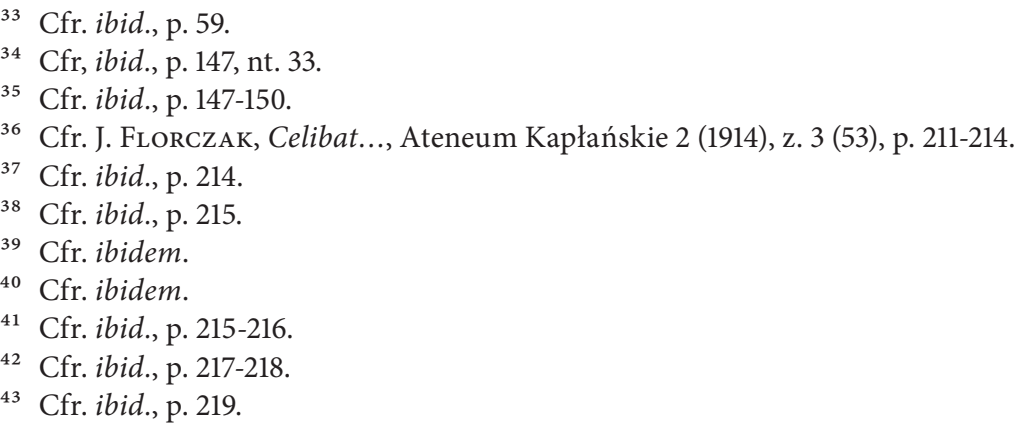


legato pontificio Ugo, cardinale del titolo di santa Sabina, vennero scomunicati i chierici concubinarii manifesti ${ }^{44}$.

Il cardinale Ugo, cistercense, del titolo di S. Lorenzo in Lucina, legato di Clemente IV (1265-1268), nel 1267 convocò un sinodo a Breslavia e all'articolo primo delle decisioni del sinodo furono comminate pene severe ai sacerdoti che tenevano concubine pubblicamente mentre per i vescovi che li tolleravano venne stabilita la sospensione $e^{45}$.

Il legato pontificio Filippo, vecovo di Fermo, convocò a Buda i vescovi ungheresi e polacchi per un sinodo, che si espresse all'art. XIV contro gli ecclesiastici dissoluti, stabilendo che essi allontanassero le loro concubine ${ }^{46}$.

L'arcivescovo di Gniezno, Jakub Świnka, il 6 gennaio 1285 riunì un sinodo provinciale a Łęczyca, che prese decisioni assai severe contro la violazione del celibato, come, ad esempio, il divieto di ascoltare la Messa celebrata da un prete sposato oppure da un prete che non viveva onestamente ${ }^{47}$. Questo sinodo ordinò ad ogni vescovo di inviare uno o due uomini credibili e giurati in tutte le chiese della propria diocesi con il potere di imprigionare le donne degli ecclesiastici, anche con l'aiuto del braccio secolare, dei padroni o del popolo $^{48}$. Il Florczak osserva come le delibere del suddetto sinodo furono approvate dai vescovi polacchi, presieduti dall'arcivescovo di Gniezno, senza alcuna pressione dei legati pontifici ${ }^{49}$ e ritorna sulla infondatezza dell'ipotesi di vescovi sposati ${ }^{50}$.

Nel terzo articolo Florczak affronta il complesso periodo della diffusione delle dottrine di Lutero contro il celibato ecclesiastico.

La posizione anticelibataria dei protestanti non trovò terreno favorevole in Polonia, sia a motivo dello zelo dei vescovi, che con

\footnotetext{
${ }^{44}$ Cfr. ibidem.

45 Cfr. ibid., p. 220.

${ }^{46}$ Cfr. ibid., p. 220-221.

${ }^{47}$ Cfr. ibid., p. 221.

${ }^{48}$ Cfr. ibidem.

49 Cfr. ibidem.

50 Cfr. ibid., p. 22-224.
} 
sinodi provinciali e diocesani avevano provveduto alla disciplina ecclesiastica, sia per la buona moralità del clero ${ }^{51}$. In Polonia furono pubblicati due libri, uno nel 1504 e l'altro nel 1516, che difendevano la possibilità di sposarsi per i preti, ma essi non ebbero un'influenza significativa, perché in quel tempo il comportamento della maggior parte del clero polacco era moralmente ineccepibile ${ }^{52}$. Verso la metà del sec. XVI in Polonia ci fu però un abbassamento nella condotta morale del clero $^{53}$, ma, osserva Florczak, bisogna riconoscere che molti autori di quel periodo, come Stanisław Orzechowski, Mikołaj Rey, Sylvester Czecanovius, Andrzej Frycz Modrzewski, Hieronim Mancelius, Hieronim Małecki (Maeletius), contagiati da princìi anticattolici, esageravano nella presentazione dell'immoralità del clero per pretendere l'abolizione del celibato ecclesiastico ${ }^{54}$.

Il celibato non veniva combattuto solo con gli scritti; ci furono infatti abbandoni del celibato da parte di religiosi comuni, ma anche di personaggi di rilievo nella Chiesa ${ }^{55}$, quali Albrecht, Grande Maestro dell'Ordine Teutonico, il vescovo di Sambia, Georg von Ponenz, già segretario di Giulio II (1503-1513), Erhard von Queis, vescovo di Pomerania, Jan Łaski, nipote del primate e nominato vescovo di Weszprim in Ungheria, Stanisław Lutomirski, canonico a Przemyśl e segretario di Augusto Sigismondo ${ }^{56}$. Questo influsso negativo proveniva dalla Prussia e si diffuse rapidamente, così che non c'era una settimana in cui non si fosse sposato un parroco, oppure un monaco o una suora ${ }^{57}$. Grande clamore suscitò il matrimonio di Franciszek Lizamnin, confessore della regina Bona, che, dopo aver abbandonato il saio, passò al calvinismo ${ }^{58}$.

\footnotetext{
51 Cfr. J. Florczak, Celibat..., Ateneum Kapłańkie 2 (1914), z. 6 (56), p. 18-19.

52 Cfr., ibid., p. 19.

53 Cfr. ibidem.

${ }^{54}$ Cfr. ibid., p. 19-21.

55 Cfr. ibid., p. 22.

${ }^{56}$ Cfr. ibid., p. 22-24.

57 Cfr. ibid., p. 23.

58 Cfr. ibid., p. 24-27.
} 
Una triste influenza ebbe in Polonia il matrimonio di Andrzej Dudycz, vescovo di Pécs in Ungheria, che l'imperatore Massimiliano II aveva inviato in Polonia, perché riconciliasse Augusto Sigismondo con la moglie ${ }^{59}$.

Nefasta fu l'azione di Stanisław Orzechowski, uomo di grande capacità, che aveva un'enorme influenza sui nobili e che nel 1547, oltre a sposarsi, scrisse una lettera nella quale attaccava con virulenza il celibato ecclesiastico, pensando di influire sul Concilio di Trento. Questo scritto, per decisione di Jan Dziaduski, vescovo di Przemyśl, fu pubblicamente bruciato $^{60}$.

Di fronte a questa situazione il nunzio in Polonia, il vescovo Kameryński, il 29 agosto 1560 scrisse al Card. Maroni, denunziando la triste situazione in cui si trovava la Polonia, perché monaci e sacerdoti prendevano moglie, senza che nessuno lo vietasse loro ${ }^{61}$.

Ma accanto a queste ombre nella Chiesa polacca brillavano anche grandi luci, sia perché, come osserva Florczak, c'erano sacerdoti che con la loro umile e piena dedizione e abnegazione rappresentavano Cristo sacerdote, sia perché ci furono autorevoli scritti a favore del celibato $^{62}$. Ad esempio, nel 1564 Marcin Kromer, poi vescovo di Warmia, pubblicò un libro a difesa del celibato, respingendo con garbo le tesi difese da Orzechowski, il quale rispose con insulti. L'opera di Kromer fu lodata da Pio IV (1560-1565) con uno speciale breve pubblicato il 9 marzo $1565^{63}$. Combattivo difensore del celibato ecclesiastico con gli scritti e con le decisioni fu Stanisław Hozjusz (Hosius), prima vescovo di Chełm e poi di Warmia e cardinale, definito "Anima della restaurazione cattolica in Polonia" ${ }^{64}$. Stanisław Zaborowski, canonico di Sandomierz e professore a Cracovia, che godeva in Polonia grande

59 Cfr. ibid., p. 25-26.

${ }^{60}$ Cfr. ibid., p. 26.

${ }^{61}$ Cfr. ibid., p. 28.

${ }^{62}$ Cfr. ibidem.

${ }^{63}$ Cfr. ibid., p. 28-29.

${ }^{64}$ I. Rogger, voce Hosius (Hos, Hozjusz Osio), Stanislao, in Enciclopedia Cattolica, vol. VI, col. 1483. 
stima per il suo dotto insegnamento, nel 1528 pubblicò un libretto dal titolo "De coelibatu christianorum et praesertim sacerdotum"65.

Sull'esempio di altri monarchi, anche Augusto Sigismondo si manifestò favorevole all'abolizione del celibato; nel 1556 inviò a Roma Stanisław Maciejowski, perché presentasse gli auguri a Paolo IV in occasione della sua elezione a Pontefice, ma anche perché manifestasse l'opportunità di abolire il celibato, in quanto in tal modo si sarebbe ottenuta la pace interna, facendo concessioni ai dissidenti ${ }^{66}$. Ma Paolo IV il 18 gennaio 1562 fece riprendere il Concilio di Trento, che, nonostante tante insistenze ed invadenti pretese perché la legge sul celibato ecclesiastico fosse cambiata, ribadì la disciplina che obbliga i chierici al celibato; inoltre nella XXV sessione vennero aggravate le pene per le trasgressioni del celibato da parte dei chierici ${ }^{67}$.

Pio IV inviò al nunzio in Polonia, Commendoni, il libro delle norme del Concilio di Trento, ordinandogli di attivarsi perché le norme fossero approvate dal re. Dopo essersi consigliato con il Card. Hozjusz, il Commendoni si recò a Parczew, dove il re aveva convocato il parlamento. L'8 agosto 1564 con un lungo ed eccellente discorso il nunzio espose le ragioni per l'accoglienza delle norme tridentine, ma il primate Jakub Uchański consiglió di leggere il libro, prima di approvarlo, attirandosi però lo sdegno dei vescovi, e così Augusto Sigismondo, senza ascoltare opinioni contrarie, approvò il libro delle norme del Concilio di Trento ${ }^{68}$. Uchański pensò di convocare un sinodo nazionale, ma i suoi sforzi sono rimasti senza esito ${ }^{69}$. Le decisioni, pur accolte, non sono state messe in atto facilmente. Il 19 maggio 1577 il sinodo convocato a Piotrków e presieduto da nunzio apostolico Vincenzo Lauro, vescovo di Mondovì, e dal primate Uchański approvò solennemente le decisioni del Concilio di Trento,

${ }^{65}$ Cfr. J. Florczak, Celibat..., Ateneum Kapłańkie 2 (1914), z. 6 (56), p. 30.

${ }^{66}$ Cfr. ibidem.

${ }^{67}$ Cfr. ibid., p. 32-33.

${ }^{68}$ Cfr. ibid., p. 33.

${ }^{69}$ Cfr. ibid., p. 33-34. 
che vennero stampate nel $1578^{70}$. Il sinodo di Piotrków, applicando il Concilio di Trento, stabilì che i matrimoni contratti da chierici erano invalidi ed inflisse pene severe contro gli ecclesiastici che contraevano il matrimonio e contro i vescovi che li tolleravano ${ }^{71}$. Dal sinodo di Piotrków fino alla fine del XVIII secolo in Polonia non si manifestò alcuna tendenza favorevole all'abolizione del celibato ecclesiastico.

Alla fine del XVIII secolo, sotto l'influenza del razionalismo proveniente dall'Occidente, incominciarono a diffondersi posizioni contrarie al celibato; c'erano ecclesiastici che appartenevano a logge massoniche, e numerosi erano quelli che si lasciavano convincere dalla filosofia di Voltaire ${ }^{72}$. Così nel 1782 venne ristampata in polacco e in latino la lettera di Stanisław Orzechowki a Giulio III (1550-1555), in cui si sosteneva l'abolizione del celibato, e venne divulgata nel paese. In quel tempo appariva anche un'altra opera contraria al celibato dal titolo "La voce dell'amante del popolo e dell'umanità alla nazione", ma ad essa fu data una risposta per sostenere il celibato del clero, fondandosi sulla Scrittura, sui Concili, sui Padri della Chiesa ed anche sulla scienza ${ }^{73}$. Anche altre opere furono scritte in favore del celibato. Ad esempio, Teofil Caroli (1739-1825) pubblicò l'opera "Vetus et constans in ecclesia catholica de sacerdotum coelibatu doctrina" e venne tradotto un libro di Jan Bl. Wilm Gratine (m. 1799) a difesa del celibato ecclesiastico ${ }^{74}$.

È da notare tuttavia anche un altro fenomeno: molti religiosi e sacerdoti diocesani abbandonavano i conventi ed il ministero, oppure chiedevano la dispensa dagli obblighi del celibato $^{75}$. All'inizio del 1800 anche tra i vescovi qualcuno si espresse a favore della libertà nei confronti del celibato; di questa idea era Siestrzencewicz, vescovo di Mohylew, in Russia, che tuttavia nei confronti del rev. Maciejowski,

\footnotetext{
Cfr. ibid., p. 34.

Cfr. ibid., p. 34.

Cfr. ibid., p. 35.

3 Cfr. ibid., p. 36

74 Cfr. ibid., p. 37.

75 Cfr. ibid., p. 35-36.
} 
direttore del ginnasio di Białystok, stimato pedagogista, che si era sposato, applicò la legge ecclesiastica, anche se con benevolenza ${ }^{76}$.

L'arcivescovo Raczyński con lettere indirizzate al re il 28 settembre 1808 e il 19 gennaio 1809 chiedeva che i sacerdoti, che si erano sposati, fossero allontanati da ogni incarico civile. La stessa richiesta al re venne fatta dai vescovi polacchi il 3 gennaio 1809 , e, poiché non venivano assecondati, i vescovi nel 1811 consegnarono al re un memoriale, con il quale chiedevano che i matrimoni contratti da ecclesiastici non fossero considerati validi agli effetti civili, ma anche questa volta la richiesta dell'episcopato rimase inesaudita ${ }^{77}$. Da quel momento la questione del celibato in Polonia non fu espressamente discussa, anche se nel 1831 una casa editrice progressista riprese l'idea di abolire il celibato, ma questa tesi non trovò sostegno ${ }^{78}$. Nel periodo in cui Pio IX (1846-1878) indisse il Concilio Vaticano I, nel 1870 contro il celibato comparve in Polonia un libro tradotto dal tedesco, che ripresentava in modo disgustoso i vecchi motivi, ma la stampa rimase indifferente. In quel tempo pochi ecclesiastici polacchi violarono il celibato ${ }^{79}$.

La difesa del celibato ecclesiastico in Polonia si deve soprattutto ad un'opera pubblicata nel 1860 da J. Ch. Janiszewski, quando era ancora parroco di Kościelec, con la quale si dimostravano il legame tra celibato e sacerdozio e la ininterrotta tradizione della Chiesa nel sostenere la legge del celibato. Dopo che era diventato vescovo ausiliare di Poznań, Janiszewski, nel 1875, mentre si trovava in carcere soffrendo per la libertà della Chiesa, pubblicò la seconda parte della sua opera ${ }^{80}$.

Quando il 19 marzo 1904 con il Motu proprio Arduum sane mu$n u s^{81}$ Pio X dichiarò di voler riformare la legislazione ecclesiastica, riprese nella stampa occidentale qualche voce a favore dell'abolizione

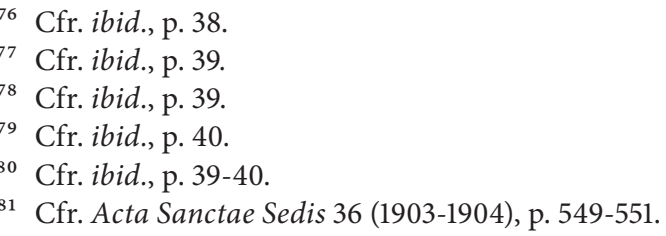


del celibato, che in Polonia però non fu assolutamente recepita, e anzi vennero pubblicate due opere a difesa del celibato: nel $1904 \mathrm{fu}$ stampato a Vilnius il libro del sacerdote J. Żuchowski, già medico, che provava come il celibato non fosse dannoso per la salute, e nel 1911 comparve un'opera del rev. J. Vaughan, tradotta dall'inglese, che, sotto forma di romanzo, intendeva dimostrare con considerazioni di carattere psicologico come il matrimonio non può favorire l'attività apostolica del sacerdote ${ }^{82}$.

Florczak, concludendo il suo studio, riferisce con amarezza che alcuni sacerdoti polacchi passavano al protestantesimo per potersi sposare, ma, dopo aver ricordato che questi erano pochi, egli afferma che il principio del celibato in Polonia continuava ad essere intoccabile ${ }^{83}$.

\section{Józef Florczak: Prelate Auditor of the Roman Rota, died in Dachau Concentration Camp}

\section{Summary}

Monsignor Józef Florczak (1885-1943) exercised his priestly ministry in a variety of positions: he was Professor and Dean of the Faculty of Canon Law and Moral Sciences of the University of Lublin, Rector of the Polish National Parish of Saint Stanislaus in Rome, Prelate Auditor of the Roman Rota and Parish Priest. He died in the Dachau concentration camp on the $20^{\text {th }}$ of April 1943.

Although his published academic work certainly is not abundant, nonetheless the attention he paid to footnotes clearly shows its scientific value.

\footnotetext{
82 Cfr. J. Florczak, Celibat..., Ateneum Kapłańkie 2(1914), z. 6(56), p. 41.

${ }^{83}$ Cfr. ibidem. Sulla storia del celibato ecclesiastico cfr. E. Jombart, v. Célibat des clercs (Droit Occidental), in Dictionnaire de Droit Canonique, sous la direction de R. Naz, t. 3, Paris 1942, col. 132-145; M. Scaduto, v. Celibato ecclesiastico, in Enciclopedia Cattolica, vol. III, col. 1262-1263; C. CochInI, Origini apostoliche del celibato ecclesiastico, tr. dal francese, Nova Millennium Romae, Roma 2011; A. M. STICKLER, Il celibato ecclesiastico. La sua storia e i suoi fondamenti teologici, Ius Ecclesiae 5 (1993), p. 3-59 (l'articolo è stato edito come volume da LEV, Città del Vaticano 1994).
} 
But where his qualities as a canonist and his wisdom as a judge are most evident, is in the thirty six sentences that Monsignor Florczak wrote as Prelate Auditor of the Roman Rota. The pars in iure of each judgement reveals his great erudition in the areas of Roman Law, theology, canonical tradition, the praxis of Roman Congregations, and of Rotal jurisprudence. In examining the sentences coram Florczak, it seemed preferable to study them according to their varied topics and thus determine their importance. To this end, the main arguments of the treatise are those that concern the different aspects of the nullity of marriage. Other specific arguments are: the invalidity of assuming the onera sacrae ordinationis, a quaestio incidens super contumacia, the competence of tribunals and the restitutio in integrum. 